\title{
Insuficiencia cardiaca por mixoma auricular izquierdo gigante: reporte de un caso
}

Giuston Mendoza-Chuctaya ${ }^{1, a}$, Alexander Montesinos-Cárdenas ${ }^{2, b}$

\section{RESUMEN}

Los mixomas son tumores cardiacos frecuentes que se localizan en su mayoría en la aurícula izquierda. Los síntomas y signos con los que se presenta son muy variados e inespecíficos en dependencia de su comportamiento. Reportamos el caso de un paciente de sexo femenino de 41 años con un tiempo de enfermedad de aproximadamente 3 meses, con cuadro clínico sugerente de insuficiencia cardiaca que se caracterizó por presentar dificultad respiratoria al reposo, dolor torácico, malestar general y edemas en miembros inferiores, el EKG sugerente de crecimiento auricular izquierdo, donde el ecocardiograma transtorácico fue prueba clave para el diagnóstico de mixoma cardiaco que abarcaba en su totalidad la aurícula izquierda, que posteriormente fue extirpado por cirugía y confirmado por anatomía patológica.

Palabras clave: Ecocardiografía; estenosis; mixoma; tumores; válvula mitral.

\section{Heart failure due to giant left atrial myxoma: a case report}

\section{ABSTRACT}

Myxomas are frequent cardiac tumors that are mostly located in the left atrium. Their signs and symptoms are very varied and nonspecific, and depend on their behavior. We report the case of a 41-year-old female patient with a disease period of approximately 3 months, and a clinical picture suggesting heart failure characterized by respiratory difficulty at rest, chest pain, general malaise and edema on lower limbs. The EKG suggested left atrial growth, where transthoracic echocardiography was a key test for the diagnosis of a myxoma filling the entire left atrium, which was later excised by surgery and confirmed by pathological anatomy.

Keywords: Echocardiogram; stenosis; myxoma; tumors; mitral valve.

1. Escuela de Medicina, Universidad Nacional de San Antonio Abad del Cusco. Cusco, Perú.

2. Hospital Adolfo Guevara Velasco, EsSalud. Cusco, Perú.

a. Estudiante de Medicina.

b. Médico Cardiólogo. 


\section{INTRODUCCIÓN}

Los tumores cardiacos primarios son raros en la práctica médica, se describen en diferentes rangos de edad pero más frecuentemente en mujeres entre los 40 a 60 años con una incidencia estimada de $0,002 \%^{(1)}$, generalmente son únicos y localizados en la aurícula izquierda unido al tabique interauricular a la altura de la fosa oval ${ }^{(2)}$. El Mixoma Auricular es un tumor histológicamente benigno que puede manifestarse con signos y síntomas clínicos variables, simulando a veces enfermedades sistémicas ${ }^{(3)}$ y alteraciones en la hemodinámica del corazón cuando obstruyen el tracto de entrada o el tracto de salida del ventrículo izquierdo, lo cual dificulta y retrasa su diagnóstico ${ }^{(1,4)}$, desde el punto de vista anatómico pueden ser encontrados en un $75 \%$ en la aurícula izquierda, un $20 \%$ en la aurícula derecha y el resto en los ventrículos, en la aurícula izquierda se encuentran frecuentemente adosados a la fosa oval, a la pared posterior o a la valva anterior de la mitral ${ }^{(1,5)}$.

\section{CASO CLÍNICO}

Paciente mujer de 41 años de edad, natural de PunoPerú, con un tiempo de enfermedad de 3 meses de inicio insidioso y progresivo, que curso con dificultad respiratoria a pequeños esfuerzos, dolor toráxico atípico y edema en miembros inferiores, por lo cual acude al hospital por emergencia, al examen físico, 24 respiraciones por minuto, 90 latidos por minuto, Presión Arterial $90 / 60 \mathrm{mmHg}$, se palpa choque de punta 5to espacio intercostal línea media clavicular, edema de miembros inferiores ++/++++, cuadro clínico de insuficiencia cardiaca descompensada NYHA III (New York Heart Association), a la auscultación presencia de soplo holosistólico III/ $\mathrm{VI}$ en foco mitral, con crépitos en bases de ambos campos pulmonares, la radiografía de tórax postero-anterior fue sugerente de Neumonía (Figura 1).

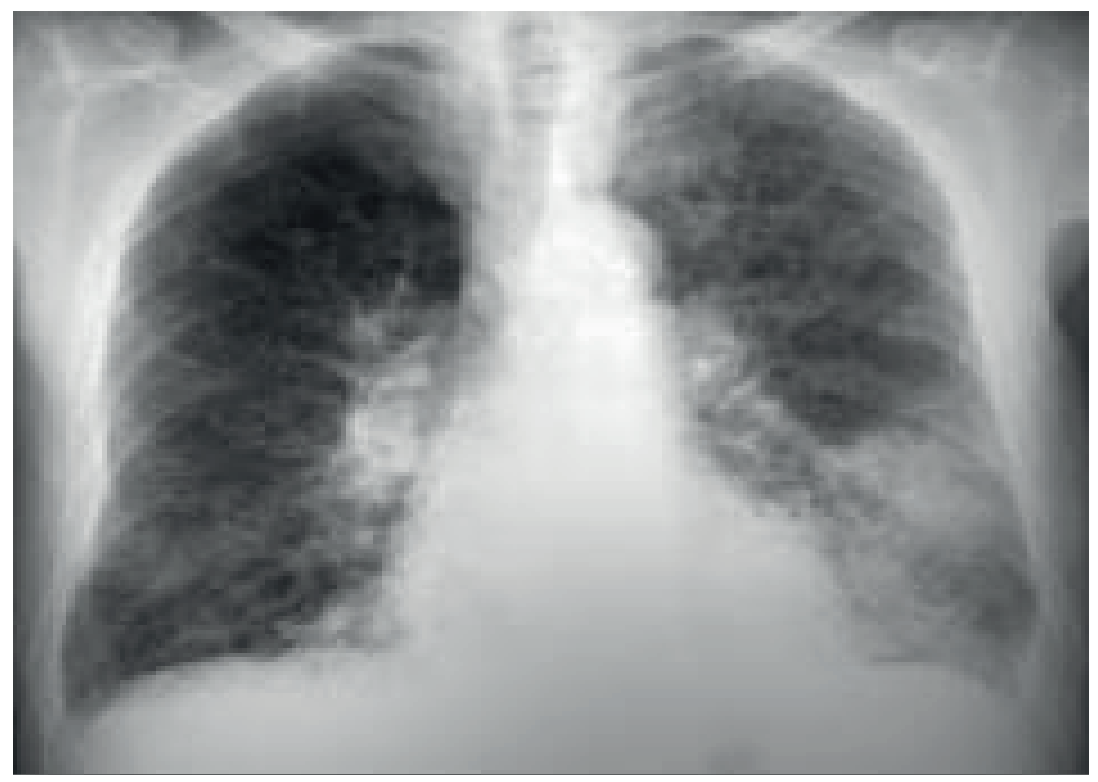

Figura 1. Se observa radiografía de tórax con infiltraciones pulmonares 
El electrocardiograma (EKG) revela ondas $\mathrm{P}$ con aumento de la prolongación y amplitud en derivación DII (Figura 2).

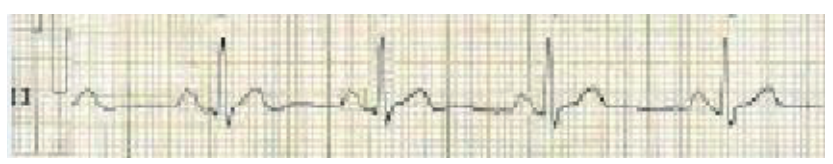

Figura 2. EKG reveló en DII ondas P con aumento de la prolongación y amplitud, criterio para crecimiento auricular izquierdo

El ecocardiograma evidenció una tumoración compatible con un mixoma que ocupaba el $100 \%$ de la aurícula izquierda en sístole ventricular, y protruía hacia el ventrículo izquierdo en diástole (Figura 3).

En la Figura 3B se aprecia el mixoma que ocupa el $100 \%$ de la aurícula izquierda en la sístole ventricular, en la Figura $3 \mathrm{~A}$ en diástole el pedículo del mixoma nace del septum interauricular y protruye hacia el ventrículo izquierdo. AD: aurícula derecha; Al: aurícula izquierda; VD: ventrículo derecho; VI: ventrículo izquierdo.

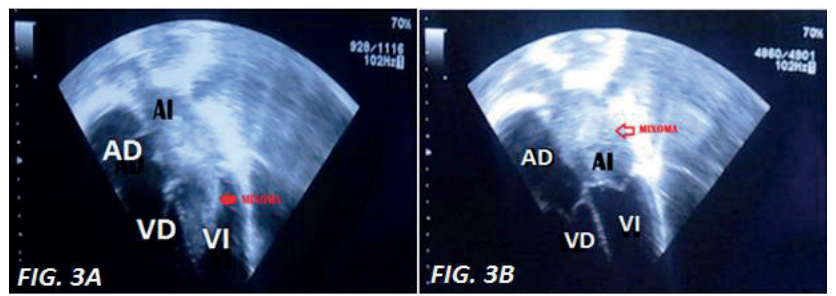

Figura 3. Imagen bidimensional, plano apical 4 cámaras

El diagnóstico diferencial sugiere descartar una posible valvulopatía mitral, hematomas intramurales, la enfermedad de Ebstein, doble lesión mitral, donde el ecocardiograma transtoráxico fue prueba clave para el diagnóstico de mixoma cardiaco que posteriormente fue extirpado por cirugía y confirmado por anatomía patológica.

\section{DISCUSIÓN}

El presente caso muestra la evolución de un tumor cardiaco primario benigno, el mixoma auricular abarcó el $100 \%$ de la aurícula izquierda ocasionando una evolución con signos y síntomas compatibles con una insuficiencia cardiaca descompensada, que son manifestaciones poco usuales, donde la ecocardiografía es prueba de elección para definir las características y localización del tumor.

Una masa en aurícula del corazón nos indica tres hipótesis diagnósticas principales: trombo auricular, tumor y vegetación, siendo la tercera fácilmente excluible por la ausencia de clínica infecciosa del paciente, en cuanto al trombo la sospecha es baja por no documentarse un sustrato protrombótico (fibrilación auricular, estenosis mitral reumática, ni miocardiopatía dilatada) y ser la aurícula una localización rara para este ${ }^{(6)}$; en cuanto al tumor cardíaco, la gran mayoría de tumores son benignos, de los cuales el mixoma representa el tumor primario benigno más común en este grupo de tumores ${ }^{(7)}$.

En los pacientes con tumoraciones benignas tipo Mixomas Auriculares, los signos y síntomas son variados e inespecíficos en dependencia de su comportamiento, pues pueden ser asintomáticos o producir efectos sistémicos, locales y fenómenos embólicos, varios autores coinciden en que las manifestaciones clínicas de los mixomas son variables, sin embargo, habitualmente pueden agruparse en: síntomas cardíacos (básicamente por obstrucción del área aurículo-ventricular), síntomas generales (fatiga, fiebre, mialgias, pérdida de peso, fenómeno de Raynaud, lesiones cutáneas) y síntomas embólicos ${ }^{(2,7,8)}$, en el presente caso se muestra una manifestación sistémica del mixoma auricular que progresivamente fue evolucionando a insuficiencia cardiaca por el gran tamaño del tumor que abarcaba completamente a aurícula izquierda, y que por las posibles complicaciones de este tipo de tumores fue extirpado mediante intervención quirúrgica de forma muy preferente ${ }^{(4,9)}$.

\section{REFERENCIAS BIBLIOGRÁFICAS}

1. Masuda I, Ferreño NM, Pasca J, Pereiro G, Lastiri H. Tumores cardíacos primarios. Mixoma auricular. Rev Fed Arg Cardiol [Internet]. 2004;33:196-204. Available from: http://www.fac. org.ar/1/revista/04v33n2/actual/act01/masuda.PDF

2. López F de Q, Anca ÁG, Huguet MR. Mixoma auricular izquierdo: presentación de dos casos. Rev Arch Médico Camagüey [Internet]. 2011;15(5):882-90. Available from: http://scielo. sld.cu/pdf/amc/v15n5/amc120511.pdf

3. Garcipérez F, Mogollón-Jiménez M, Ortiz R. Porro Insuficiencia cardiaca aguda en paciente con mixoma auricular simulando estenosis mitral severa. SEMERGEN - Medicina de Familia.2014;40(3):, 171-2

4. Enzo L G, María N P, Mario G C, Carlos V, María D. P. VO, Eduardo D, et al. Mixomas cardíacos: presentación clínica, resultados quirúrgicos y pronóstico a largo plazo. Rev argent cardiol [Internet]. 2010;78:108-13. Available from: /scielo. php?script=sci_arttext\&pid=\&lang=pt

5. Arauz A, Cantu C, Merlos-Benitez M, Hernandez-Curiel B, Barinagarrementeria F, Roldan J. Stroke as a complication of atrial myxoma. Report of six cases and review of literature. [Spanish] Enfermedad cerebrovascular como complicacion de mixoma auricular. Reporte de seis casos y revision de la literatura. Rev Mex Neurocienc [Internet]. 2010;11:1948. Available from: http://www.medigraphic.com/pdfs/ revmexneu/rmn-2010/rmn103b.pdf

6. Zamora Bastidas Tomás, Maya Ruiz Daira, Rángel Magda, López Garzón Nelson, Bermúdez Joaquín Milton Mixoma: manifestaciones neurológicas y reumatológicas. Informe de casos. Rev Urug Cardiol. 2013;28: 116-21

7. Yglesias PJM, Climente MR. Mixoma auricular izquierdo como forma de presentación de una angina de esfuerzo. An Med Interna [Internet]. 2006;23:279-81. Available from: http:// scielo.isciii.es/pdf/ami/v23n6/nota2.pdf 
8. Surabhi SK, Fasseas P, Vandecker WA, Hanau CA, Wolf NM. Right Atrial Myxoma in a Patient Presenting with Syncope. Texas Hear Inst. 2001;28(3):228-9.

9. Vinasco DMO, Sánchez MA, Osorio JE. Insuficiencia mitral severa posresección de mixoma auricular gigante : presentación de un caso y revisión de la literatura. Rev Esp Anestesiol Reanim [Internet]. 2013;60(7):403-6. Available from: http:// apps.elsevier.es/watermark/ctl_servlet?_f=10\&pident articulo=90223219\&pident_usuario=0\&pcontactid=\&pident revista=344\&ty=28\&accion=L\&origen=zonadelectura\& $w e b=w w w$. elsevier.es\&lan=es\&fichero=344v60n07a90223219pdf001.pdf

\section{Fuentes de financiamiento:}

Este artículo ha sido financiado por los autores.

\section{Conflictos de interés:}

Los autores declaran no tener ningún conflicto de interés.

\section{Correspondencia:}

Giuston Mendoza Chuctaya

Dirección: APV Praderas del Inca B-5 San Sebastián, Cusco, Perú. Teléfono: 984040426

Correo electrónico: giustonmch@gmail.com

Recibido: 31 de octubre de 2016

Aprobado: 31 de enero de 2017 